\title{
Predictive factors of symptomatic radiation pneumonitis in primary and metastatic lung tumors treated with stereotactic ablative body radiotherapy
}

\author{
Kangpyo Kim, MD, Jeongshim Lee, MD, Yeona Cho, MD, Seung Yeun Chung, MD, \\ Jason Joon Bock Lee, MD, Chang Geol Lee, MD, Jaeho Cho, MD, PhD \\ Department of Radiation Oncology, Yonsei University College of Medicine, Seoul, Korea
}

\begin{abstract}
Purpose: Although stereotactic ablative body radiotherapy (SABR) is widely used therapeutic technique, predictive factors of radiation pneumonitis (RP) after SABR remain undefined. We aimed to investigate the predictive factors affecting RP in patients with primary or metastatic lung tumors who received SABR.

Materials and Methods: From 2012 to 2015, we reviewed 59 patients with 72 primary or metastatic lung tumors treated with SABR, and performed analyses of clinical and dosimetric variables related to symptomatic RP. SABR was delivered as 45-60 Gy in 3-4 fractions, which were over $100 \mathrm{~Gy}$ in BED when the $\alpha / \beta$ value was assumed to be 10 . Tumor volume and other various dose volume factors were analyzed using median value as a cutoff value. RP was graded per the Common Terminology Criteria for Adverse Events v4.03.

Results: At the median follow-up period of 11 months, symptomatic RP was observed in 13 lesions (12 patients, 18.1\%), including grade 2 RP in 11 lesions and grade 3 in 2 lesions. Patients with planning target volume (PTV) of $\leq 14.35 \mathrm{~mL}$ had significantly lower rates of symptomatic RP when compared to others ( $8.6 \% \mathrm{vs}$. $27 \% ; p=0.048)$. Rates of symptomatic RP in patients with internal gross tumor volume (iGTV) $>4.21 \mathrm{~mL}$ were higher than with $\leq 4.21 \mathrm{~mL}(29.7 \%$ vs. $6.1 \% ; p=0.017)$.

Conclusions: The incidence of symptomatic RP following treatment with SABR was acceptable with grade 2 RP being observed in most patients. iGTV over $4.21 \mathrm{~mL}$ and PTV of over $14.35 \mathrm{~mL}$ were significant predictive factors related to symptomatic RP.
\end{abstract}

Keywords: Lung cancer, Radiation pneumonitis, Stereotactic ablative body radiotherapy, Risk factors

\section{Introduction}

Stereotactic ablative body radiotherapy (SABR) has been widely used in the treatment of early-stage primary lung cancers due to its high local control rates of approximately $90 \%$ or more [1]. In addition, SABR is also used to treat metastatic lung tumors in the oligometastatic setting as salvage therapy [2]. Along with increasing the application of SABR, we also need to evaluate the factors relating to SABR associated toxicity, especially radiation pneumonitis (RP) [3,4]. However, there are limited reports on the predictive factors for developing RP after treatment with SABR for primary or metastatic lung tumors. Previous reports showed that the incidence of RP ranges from $9 \%$ to $28 \%$, mostly of grade 2 or less [5].

Dose-volume values are potential factors that could predict the development of RP after treatment with SABR.

Received 06 February 2017, Revised 10 April 2017, Accepted 04 May 2017.

Correspondence: Jaeho Cho, MD, PhD, Department of Radiation Oncology, Yonsei University College of Medicine, 50-1 Yonsei-ro, Seodaemun-gu, Seoul 03722, Korea. Tel: +82-2-2228-8113, Fax: +82-2-2227-7823, E-mail: jjhmd@yuhs.ac

(c) This is an Open Access article distributed under the terms of the Creative Commons Attribution Non-Commercial License (http://creativecommons.org/ licenses/by-nc/4.0/) which permits unrestricted non-commercial use, distribution, and reproduction in any medium, provided the original work is properly cited.

www.e-roj.org 
In conventional fractionation, the Quantitative Analysis of Normal Tissue Effects in the Clinic (QUANTEC) recommends a $V_{20}$ of $\leq 30 \%$ to $35 \%$ and a mean lung dose (MLD) of $\leq 20$ to 23 Gy to reduce the risks of developing RP to less than $20 \%$ [6]. In some studies, size of target, $V_{20}-V_{25}$ of bilateral lung dose or MLD has been shown as a possible predictive factor of symptomatic pneumonitis [7-11]. However, there are no clear guidelines for the dose-volume limits for treatment with SABR. As tissues irradiated with high fraction doses are expected to show different radiobiologic responses, it is difficult to predict the possibility of developing RP by using traditional dosimetric guidelines [12].

In this study, we aim to determine the clinical and dosevolume factors that affect the development of symptomatic $\mathrm{RP}$ after treatment of primary or metastatic lung tumors with $S A B R$, to facilitate the application of SABR in practical settings.

\section{Materials and Methods}

\section{Study design}

This study was approved by the Yonsei University Health System Institutional Review Board (No. 4-2017-0108).

We retrospectively reviewed data on patients with primary and metastatic lung tumors who underwent treatment with SABR from March 2012 to March 2015 in our institution. Inclusion criteria were as follows: age above 20 years, histologically confirmed primary lung cancers and histologically or radiologically diagnosed as metastatic lung tumors, and an Eastern Cooperative Oncology Group (ECOG) performance status of $0-1$. For selection of eligible patients, we reviewed medical records, laboratory results, and imaging studies. Finally, we retrieved 72 tumors from 59 patients with primary lung cancers $(n=29)$ and metastatic lung tumors ( $=43$ ). The locations of these lung tumors were divided into central versus peripheral lesions, according to the Radiation Therapy Oncology Group (RTOG) 0813 protocol [13]; central lesions are defined as the tumors located at $<2 \mathrm{~cm}$ from the proximal bronchial tree, or with a planning target volume (PTV) touching the mediastinum, or both. Non-central lesions are considered as peripheral tumors. Single lesion was treated for 46 patients and 2 lesions for 13 patients. Each of 2 lesions were located in the opposite lung separately and treated with radiotherapy at the interval of 6 months or more. As SABR is characterized by steep dose fall off we decided to analyze by 72 lesions.

\section{Procedure for SABR}

All patients underwent simulation computed tomography (CT) with immobilizing devices, such as, a whole-body vacuum lock or a stereotactic body frame, and an abdominal compressor for respiration control. A shallow breathing technique using Abches (APEX Medical Inc., Tokyo, Japan) was also applicable for patients who were intolerant to the body compressor. If the respiratory diaphragm moved over $1 \mathrm{~cm}$ vertically, the abdominal compressor or shallow breath technique using Abches were indicated for LINAC multiport group and intensity-modulated radiotheray (IMRT) group. All patients underwent four-dimensional CT (4D-CT) scans to track the movement of the target along the respiratory movement. For SABR, the gross tumor volume (GTV) was delineated in every phase of the 4D-CT images, and the PTV was defined as the internal gross tumor volume (iGTV) with a 5- to $10-$ $\mathrm{mm}$ margin. In patients treated with CyberKnife (Accuray Inc. Sunnyvale, CA, USA), whole-body vacuum lock was used for the stability of patients during treatment. However abdominal compressor or Abches was not indicated as CyberKnife can track the tumor along the respiratory movement. PTV for patients treated with CyberKnife group was minimized to 2-3 $\mathrm{mm}$. Four dose schemes were used according to the tumor location and volume, and were administered as 48 Gy in 4 fractions (fx), 45 Gy in 3 fx, 60 Gy in 4 fx, and $60 \mathrm{~Gy}$ in $3 \mathrm{fx}$; these were prescribed to the volumetric PTV and the primary aim was to cover 100\% volume of the PTV with $80 \%$ isodose line of the prescribed dose. When converted to biologically equivalent dose (BED), these doses are 106 Gy, 113 Gy, 150 Gy, and $180 \mathrm{~Gy}$ when the $\alpha / \beta$ value of the tumor is assumed to be $10 \mathrm{~Gy}$. All patients were treated every other day. Specific prescriptions of SABR doses are shown in Table 1.

Different planning systems according to treatment modality are used and specific name of planning systems are as follows; Pinnacle3 treatment planning system (Philips Radiation Oncology Systems, Milpitas, CA, USA) for LINAC multiport

Table 1. Characteristics of stereotactic ablative body radiotherapy $(n=72)$

\begin{tabular}{lcccc}
\hline $\begin{array}{c}\text { Dose } \\
\text { prescription }\end{array}$ & $\begin{array}{c}\text { LINAC } \\
\text { multiport }\end{array}$ & VMAT & TomoTherapy & CyberKnife \\
\hline 4,800 cGy/4 fx & 6 & 4 & 2 & 0 \\
4,500 cGy/3 fx & 22 & 1 & 2 & 6 \\
6,000 cGy/4 fx & 6 & 5 & 3 & 0 \\
6,000 cGy/3 fx & 9 & 0 & 3 & 3 \\
\hline LINAC, linear accelerator; VMAT, volume modulated arc therapy; \\
fx, fraction.
\end{tabular}


group, RayStation (RaySearch Laboratories AB, Stockholm, Sweden) for volumetric modulated arc therapy (VMAT) group, TomoTherapy treatment planning system (Accuray Inc.) for TomoTherapy group, and Multiplan Treatment Planning System v2.05 (Accuray Inc.) for CyberKnife group.

Normal organ dose constraints for SABR are based on the report of the American Association of Physicists in Medicine Task Group 101 (AAPM TG 101) [14]. Dose constraints for specific organ are as follows: $12 \mathrm{~Gy} / 3 \mathrm{fx}$ and $13 \mathrm{~Gy} / 4 \mathrm{fx}$ for MLD, $V_{20}<10 \%$ for bilateral lung, 39 Gy for great vessel and 15 Gy for trachea and bronchial tree.

Delivery quality assurances for each patient and each radiation treatment were done before start of treatment, and the use of $4 \mathrm{D}$ cone beam CT (Symmetry) or megavoltage CT for accurate delivery were also mandatory. When tumors were treated with CyberKnife (Accuray Inc.), a tracking system was applied. As we do not insert gold fiducial markers, tumors visible on either one or both X-ray cameras were indicated for treatment with CyberKnife. Patients who could not tolerate the duration of treatment and had non-trackable lesions were excluded from the indication for treatment.

\section{Assessment of radiation pneumonitis}

Patients were assessed through regular follow-up visits, and underwent chest CT scans at 1, 3, and 6 months after undergoing treatment with SABR. Per the decision of the physician, positron emission tomography-computed tomography (PET-CT) scans were also available at 3 to 6 months after treatment with SABR, particularly for metastatic lung tumors, to confirm the extent of disease. RP was graded in 5 levels per the Common Terminology Criteria for Adverse Events (CTCAE) ver $4.03[15,16]$. RP was diagnosed based on clinical symptoms and radiologic findings, which develop within 6 months after undergoing treatment with SABR $[16,17]$. We assessed symptomatic RP that was defined as grade 2 or worse, and analyzed the predictive factors related to symptomatic RP.

\section{Data analysis and statistical method}

We measured PTV (in $\mathrm{mL}$ ), iGTV (in $\mathrm{mL}$ ), tumor diameter (in $\mathrm{cm}$ ), MLD (in Gy), bilateral $V_{51}, V_{10}, V_{15}, V_{20}, V_{25}, V_{30}, V_{35}$ and $V_{40}$ (in \%). $V_{d}$ is defined as the volume of the bilateral normal lung that received a radiation dose of over ' $d$ ' Gy. Target volumes, lung volumes, and $V_{d}$ were measured using MIM software (MIM Software Inc., Cleveland, OH, USA). Potential predictive factors for the occurrence of an RP event were: patient age, sex, the ECOG performance status, smoking history, underlying pulmonary disease, pathology, tumor location, fraction size and total dose of SABR, PTV, the number of targets, and dosimetric parameters, such as, ipsilateral and contralateral $M L D$, and $V_{5}-V_{40}$ in increments of $5 \mathrm{~Gy}$.

We used median value as the cutoff values of iGTV, PTV, tumor diameter, MLD, and $V_{d}$ for statistic analysis. Chisquare test was used for univariable analysis for all variables. Characteristics which are found to be significant and $V_{20} N_{25}$ were entered in stepwise method in a binary logistic regression analysis to develop multivariate model. SPSS ver. 20.0 (IBM, Armonk, NY, USA) was used for the analysis, and a p-value of $<0.05$ was defined as statistically significant.

\section{Results}

\section{Characteristics of patients, tumors, and treatments}

All baseline characteristics are listed in Table 2. Median duration of follow-up was 11 months (range, 6 to 31 months). Twenty-nine lesions (40.3\%) were primary lung cancers, and the others were metastatic sites (59.7\%). Forty-two lesions (58.3\%) were on the left lung, and the remaining were on the right side. Eighteen lesions (25\%) were in the central area. Forty-six patients were treated for single lesion with SABR, while 13 patients were treated for 2 lung lesions. The 2 lesions were located in the opposite lung and they were treated with SABR at intervals of more than 6 months. Median iGTV was 4.21 $\mathrm{mL}$ (range, 0.23 to $36.43 \mathrm{~mL}$ ) and the PTV was $14.35 \mathrm{~mL}$ (range, 3.33 to $78.61 \mathrm{~mL}$ ).

Median time of treatment was 7 days (range, 4 to 12 days). The individual radiation treatment schemes were as follows: 31 lesions treated with $45 \mathrm{~Gy}$ in $3 \mathrm{fx}, 12$ lesions with $48 \mathrm{~Gy}$ in $4 \mathrm{fx}, 14$ lesions with $60 \mathrm{~Gy}$ in $4 \mathrm{fx}$, and 15 lesions with $60 \mathrm{~Gy}$ in $3 \mathrm{fx}$. All irradiated doses to normal organs were acceptable, according to the report of AAPM TG 101.

\section{Incidence of symptomatic RP}

During the median follow-up period, symptomatic RP developed in 13 lesions (12 patients, 18.1\%). Of these patients, 11 had grade 2 RP and two had grade 3 RP with a median onset of 14 weeks (range, 4 to 24 weeks) from the starting date of radiotherapy. No patients showed RP of grade 4 or higher. All patients with RP over grade 2 achieved symptomatic relief with conservative management and the use of steroid agents. One patient with grade 3 RP is shown in Fig. 1 with radiologic image. 
Table 2. Characteristics of patients $(n=59)$ and tumors $(n=72)$

\begin{tabular}{|c|c|}
\hline Characteristic & Value \\
\hline Age (yr) & $63(22-86)$ \\
\hline \multicolumn{2}{|l|}{ Sex } \\
\hline Male & 40 (67.8) \\
\hline Female & $19(32.2)$ \\
\hline \multicolumn{2}{|l|}{ Site of primary cancer } \\
\hline Lung & $29(49.2)$ \\
\hline Colorectum & $15(25.4)$ \\
\hline Head and neck & $6(10.2)$ \\
\hline Hepatobiliary & $4(6.8)$ \\
\hline Genitourinary & $4(6.8)$ \\
\hline Malignant melanoma & $1(1.7)$ \\
\hline \multicolumn{2}{|c|}{$\begin{array}{l}\text { Number of treated lung lesions } \\
\text { ( } n=59 \text { patients) }\end{array}$} \\
\hline 1 & $46(78.0)$ \\
\hline 2 & $13(22.0)$ \\
\hline \multicolumn{2}{|l|}{ Smoking history } \\
\hline Yes & 33 (55.9) \\
\hline No & $26(44.1)$ \\
\hline \multicolumn{2}{|l|}{ Any pulmonary disease } \\
\hline Yes & $23(39.0)$ \\
\hline No & $36(61.0)$ \\
\hline ILD & $2(3.4)$ \\
\hline No ILD & $57(96.6)$ \\
\hline \multicolumn{2}{|l|}{ Lobes of lung } \\
\hline RUL & $13(18.1)$ \\
\hline RML & $7(9.7)$ \\
\hline RLL & $10(13.9)$ \\
\hline LUL & $24(33.3)$ \\
\hline LLL & $18(25)$ \\
\hline \multicolumn{2}{|l|}{ Location of lung } \\
\hline Central & $18(25)$ \\
\hline Peripheral & $54(75)$ \\
\hline \multicolumn{2}{|l|}{ Lung mass size profiles } \\
\hline Tumor diameter $(\mathrm{cm})$ & $2.0(0.8-4.1)$ \\
\hline iGTV $(\mathrm{mL})$ & $4.21(0.23-36.43)$ \\
\hline $\mathrm{PTV}(\mathrm{mL})$ & $14.35(3.33-78.61)$ \\
\hline
\end{tabular}

Values are presented as median (range) or number (\%). ILD, interstitial lung disease; $R U L$, right upper lobe; $R M L$, right middle lobe; $R L L$, right lower lobe; $L U L$, left upper lobe; $L L L$, left lower lobe; iGTV, internal gross tumor volume; PTV, planning target volume.

\section{Predictive factors for symptomatic RP}

The predictive factors associated with the development of RP were analyzed, and both iGTV and PTV were established as significant predictive factors. The detailed analyses of clinical factors related to symptomatic RP are shown in Table 3, and that of dosimetric factors in Table 4.

In multivariate analysis, there were significant differences in the incidence of symptomatic RP in patients with PTV of $\leq 14.35 \mathrm{~mL}$ compared to those with PTV of $>14.35 \mathrm{~mL}$ (8.6\% vs. 27\%; $p=0.048$ ). Additionally iGTV of $\leq 4.21 \mathrm{~mL}$ was significant cut off value for the reduced incidence of RP (6\% vs. 30\%; $p=0.017$ ) (Table 5). Other factors such as patient age, sex,
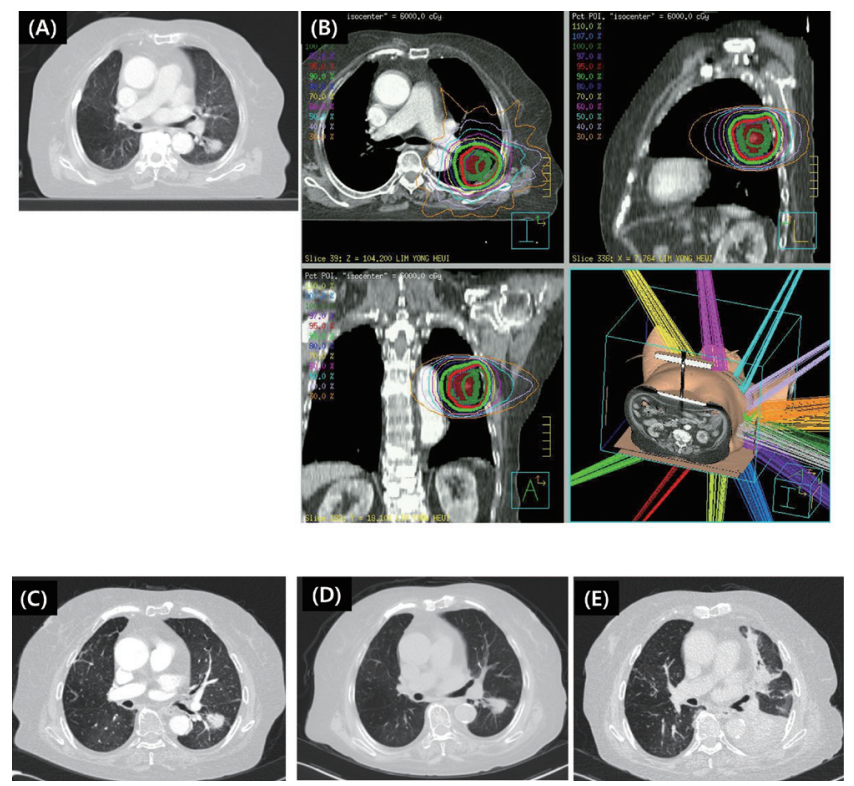

Fig. 1. An 82-year-old woman with asthma was diagnosed with adenocarcinoma of the left lower lung, which was classified as cT1bNOMO per TNM system of classification (A). Due to old age and impaired cardiac function, SABR was delivered as a total dose of $60 \mathrm{~Gy}$ in 3 fractions, as an alternative to surgery. SABR was delivered with 14 non-coplanar beams to the lesion in the left lower lung (B). She underwent a CT scan of the chest at 1 month (C) and 3 months (D) after undergoing treatment with SABR. She had no complaints, and both CT scans showed no abnormal signs related to radiation pneumonitis. However, after 5 months of SABR, she developed dyspnea, and underwent a CT scan of her chest for the evaluation of dyspnea. Newly developed pleural effusion with consolidation was observed, and diagnosed as radiation pneumonitis of grade 3 (E). SABR, stereotactic ablative body radiotherapy; $\mathrm{CT}$, computed tomography.

the ECOG performance status, smoking history, underlying pulmonary disease, pathology, tumor location, fraction size and total dose of SABR, MLD, the number of treated targets and tumor diameter did not show association with the development of symptomatic RP. Dosimetric factors, including $V_{5}, V_{10}, V_{15}, V_{20}, V_{25}, V_{30}, V_{35}$, and $V_{40}$, were not significant predictive factors that influenced symptomatic RP.

The incidence of pneumonitis between patients with single lesion and 2 lesions was not different (21.7\% vs.11.5\%; $p=$ 0.352).

\section{Dose-volume values and the incidence of RP according} to the radiation treatment modalities

We analyzed the incidence of symptomatic RP and dosevolume factors according to the 4 treatment modalities: the 
Table 3. Predictive factor univariate analysis of symptomatic RP

\begin{tabular}{|c|c|c|c|}
\hline Variable & $\mathrm{RP}$ & No RP & $p$-value \\
\hline Sex & & & 0.476 \\
\hline Male & $9(22.5)$ & $31(77.5)$ & \\
\hline Female & $3(15.8)$ & $16(84.2)$ & \\
\hline Smoking history & & & 0.488 \\
\hline Yes & $8(24.2)$ & $25(75.8)$ & \\
\hline No & $4(15.4)$ & $22(84.6)$ & \\
\hline Any pulmonary history & & & 0.725 \\
\hline Yes & $4(17.4)$ & $19(82.6)$ & \\
\hline No & $8(22.2)$ & $28(77.8)$ & \\
\hline Lung site & & & 0.325 \\
\hline Right & 7 (23.3) & 23 (66.7) & \\
\hline Left & $6(14.3)$ & $36(85.7)$ & \\
\hline Location & & & 0.860 \\
\hline Central & 3 (16.7) & 15 (83.3) & \\
\hline Peripheral & $10(18.5)$ & $44(81.5)$ & \\
\hline Dose schemes & & & 0.583 \\
\hline 4,500 cGy/3 fx & $8(25.8)$ & $23(74.2)$ & \\
\hline $4,800 \mathrm{cGy} / 4 \mathrm{fx}$ & $1(8.3)$ & $11(91.7)$ & \\
\hline $6,000 \mathrm{cGy} / 4 \mathrm{fx}$ & $2(14.3)$ & $12(85.7)$ & \\
\hline $6,000 \mathrm{cGy} / 3 \mathrm{fx}$ & $2(13.3)$ & $13(86.7)$ & \\
\hline Mean lung dose (Gy) & & & 0.541 \\
\hline$<3.2$ & $5(13.9)$ & $31(86.1)$ & \\
\hline$\geq 3.2$ & $8(22.2)$ & $28(77.8)$ & \\
\hline iGTV (mL) & & & 0.013 \\
\hline$\leq 4.21$ & $2(6.1)$ & 34 (93.9) & \\
\hline$>4.21$ & $11(29.7)$ & $25(70.3)$ & \\
\hline PTV (mL) & & & 0.042 \\
\hline$\leq 14.35$ & 3 (8.6) & 33 (91.4) & \\
\hline$>14.35$ & $10(27.0)$ & $26(73.0)$ & \\
\hline Tumor diameter (cm) & & & 0.131 \\
\hline$\leq 2.0$ & $4(11.1)$ & 32 (88.9) & \\
\hline$>2.0$ & $9(25.0)$ & $27(75.0)$ & \\
\hline Number of treated lung lesions & & & 0.352 \\
\hline 1 & $10(21.7)$ & $36(78.3)$ & \\
\hline 2 & $3(11.5)$ & 23 (88.5) & \\
\hline Treatment modality & & & 0.138 \\
\hline LINAC multiport & $11(25.6)$ & $32(74.4)$ & \\
\hline $\begin{array}{l}\text { IMRT } \\
\text { (VMAT and TomoTherapy) }\end{array}$ & $2(10)$ & $18(90)$ & \\
\hline CyberKnife & $0(0)$ & $9(100)$ & \\
\hline
\end{tabular}

Values are presented as number (\%).

$\mathrm{RP}$, radiation pneumonitis; $\mathrm{fx}$, fraction; iGTV, internal gross tumor volume; PTV, planning target volume; LINAC, linear accelerator; IMRT, intensity-modulated radiotherapy; VMAT, volumetric modulated arc therapy.

LINAC multiport, VMAT, TomoTherapy, and CyberKnife with tumor tracking system. There was no difference of incidence of symptomatic RP and dosimetric values between modalities. No patients who were treated with CyberKnife suffered from symptomatic RP, although the PTV, MLD, and $V_{5}-V_{40}$ in this group were not significantly different from the LINAC multiport group.
Table 4. Predictive factor univariate analyses of symptomatic RP according to $V_{5}-V_{40}$ of bilateral normal lung parenchyme $(n=72)$

\begin{tabular}{|c|c|c|c|}
\hline Variable & $\mathrm{RP}$ & No RP & $p$-value \\
\hline$V_{5}(\%)$ & & & 1.0 \\
\hline$<15.00$ & 6 (16.7) & 30 (83.3) & \\
\hline$\geq 15.00$ & 7 (19.4) & 29 (80.6) & \\
\hline$V_{10}(\%)$ & & & 0.541 \\
\hline$<8.76$ & 5 (13.9) & 31 (86.1) & \\
\hline$\geq 8.76$ & $8(22.2)$ & $28(77.8)$ & \\
\hline$V_{15}(\%)$ & & & 0.541 \\
\hline$<5.27$ & $5(13.9)$ & 31 (86.1) & \\
\hline$\geq 5.27$ & $8(22.2)$ & 28 (77.8) & \\
\hline$V_{20}(\%)$ & & & 0.222 \\
\hline$<3.56$ & $4(11.1)$ & 32 (88.9) & \\
\hline$\geq 3.56$ & $9(25.0)$ & 27 (75.0) & \\
\hline$V_{25}(\%)$ & & & 0.222 \\
\hline$<2.58$ & $4(11.1)$ & 32 (88.9) & \\
\hline$\geq 2.58$ & $9(25.0)$ & $27(75.0)$ & \\
\hline$V_{30}(\%)$ & & & 1.0 \\
\hline$<1.78$ & 6 (16.7) & 30 (83.3) & \\
\hline$\geq 1.78$ & 7 (19.4) & 29 (80.6) & \\
\hline$V_{35}(\%)$ & & & 1.0 \\
\hline$<1.33$ & $6(16.7)$ & 30 (83.3) & \\
\hline$\geq 1.33$ & $7(19.4)$ & 29 (80.6) & \\
\hline$V_{40}(\%)$ & & & 1.0 \\
\hline$<1.04$ & $6(16.7)$ & 30 (83.3) & \\
\hline$\geq 1.04$ & $7(19.4)$ & 29 (80.6) & \\
\hline
\end{tabular}

Values are presented as number (\%).

$\mathrm{RP}$, radiation pneumonitis; $\mathrm{V}_{d}(\%)$, volume of bilateral normal lung that received a dose of over 'd' Gy.

\section{Discussion and Conclusion}

Acute RP is a common side effect of radiation treatment that can significantly affect the quality of life of patients, by inducing chronic respiratory problems and sometimes death, therefore, many studies have reported the predictive factors related to RP [18-21]. However, in SABR or SBRT, there is no clear consensus for predicting the risk of RP in relation to the clinical and dosimetric factors. In this study, we showed that a value of iGTV of over $4.21 \mathrm{~mL}$ and PTV of over $14.35 \mathrm{~mL}$ were significant factors for developing acute symptomatic RP after treatment of primary lung cancers and metastatic lung tumors with SABR.

Some recent studies have suggested predictive factors of RP after treatment with SABR for lung tumors. We have summarized previous studies that dealt with the dose-volume factors related to RP in Table 6. A group from the Netherlands Vrije University reported that almost three times the incidence of symptomatic RP (10\% vs. $28 \%$ ) appeared after treatment with SABR when the PTV was over $80 \mathrm{~cm}^{3}$ [22]. In 251 patients of node negative stage I-IIB non-small cell lung cancers (NSCLCs), Barriger et al. [7] reported predictive factors of grade 
Table 5. Multivariate analysis of predictive factor analyses of symptomatic RP $(n=72)$

\begin{tabular}{|c|c|c|c|}
\hline Variable & $\mathrm{RP}$ & No RP & $p$-value \\
\hline Tumor diameter $(\mathrm{cm})$ & & & 0.342 \\
\hline$\leq 2.0$ & $4(11.1)$ & 32 (88.9) & \\
\hline$>2.0$ & $9(25.0)$ & $27(75.0)$ & \\
\hline iGTV (mL) & & & 0.017 \\
\hline$\leq 4.21$ & $2(6.1)$ & $34(93.9)$ & \\
\hline$>4.21$ & 11 (29.7) & 25 (70.3) & \\
\hline PTV (mL) & & & 0.048 \\
\hline$\leq 14.35$ & 3 (8.6) & 33 (91.4) & \\
\hline$>14.35$ & $10(27.0)$ & $26(73.0)$ & \\
\hline Mean lung dose (Gy) & & & 0.754 \\
\hline$<3.2$ & $5(13.9)$ & $31(86.1)$ & \\
\hline$\geq 3.2$ & $8(22.2)$ & $28(77.8)$ & \\
\hline$V_{20}(\%)$ & & & 0.342 \\
\hline$<3.56$ & $4(11.1)$ & 32 (88.9) & \\
\hline$\geq 3.56$ & $9(25.0)$ & $27(75.0)$ & \\
\hline$V_{25}(\%)$ & & & 0.342 \\
\hline$<2.58$ & $4(11.1)$ & 32 (88.9) & \\
\hline$\geq 2.58$ & $9(25.0)$ & $27(75.0)$ & \\
\hline Treatment modality & & & 0.416 \\
\hline LINAC multiport & $11(25.6)$ & $32(74.4)$ & \\
\hline IMRT (VMAT, TomoTherapy) & $2(10)$ & $18(90)$ & \\
\hline CyberKnife & $0(0)$ & $9(100)$ & \\
\hline
\end{tabular}

Values are presented as number (\%).

$\mathrm{RP}$, radiation pneumonitis; iGTV, internal gross tumor volume; PTV, planning target volume; $V_{d}(\%)$, volume of bilateral normal lung that received a dose of over 'd' Gy; LINAC, linear accelerator; IMRT, intensity-modulated radiotherapy; VMAT, volumetric modulated arc therapy.

$\geq 2$ RP when treated with SABR. In this study, MLD >4 Gy and V20 >4\% were significant factors for RP, although PTV was not a significant risk factor for the development of symptomatic RP. In addition, Matsuo et al. [5] showed that PTV, V20, and V25 were independent predictive factors of symptomatic RP for patients diagnosed with stage I NSCLCs who received SABR with $48 \mathrm{~Gy}$ in 4 fractions. Interestingly, there was a study of Ong et al. [23] reporting that contralateral lung V5 significantly affect the development of symptomatic RP. Comparted to other reported literatures in Table 6, crude rate of $18.1 \%$ of grade $\geq 2$ pneumonitis in our study is somewhat higher. This can be explained by a strict symptom control in our clinic. All patients presenting clinical symptoms with suspicious radiologic changes at follow-up are recommended to use steroid agents and this might have resultes in an overestimation of the incidence. In agreement with other studies, there were limited incidence of grade $>2$ pneumonitis in our study also; two cases of grade 3 RP and no grade 4 or 5 .

In our study, only tumor and target size were significant factors related to symptomatic RP. Meaning of other dose volume factors such as MLD and $V_{5}-V_{40}(\%)$ might have been diluted as the prescription dose and fraction number were different for each lesion treatment modality was not a significant predictive factor for RP. PTV size between LINAC multiport group and IMRT group was significantly different (20.0 $\mathrm{mL}$ vs. $11.7 \mathrm{~mL} ; \mathrm{p}=0.019$ ) and this is the reason that more people suffered symptomatic RP in LINAC multiport group, without significance (25.6\% vs. $10.9 \% ; p=0.19)$.

As we mentioned above, many studies failed to suggest common dose volume factors of cutoff values which are critical to the development of RP. Thus, in general, both the volume reduction of the target (significant predictive factor in our study) and the dose restriction for bilateral lungs after treatment of lung tumors with SABR is recommended. In practice, based on other reports including those of our study, most radiation oncologists are trying to reduce the PTV and irradiated dose to normal lungs in many aspects. Firstly, 4DCT simulation is gaining popularity for targeting moving organs, especially the lungs. The 4D-CT leads to 10 phases of images per the respiration cycle, and it reflects the movement of the target organs. These moving images may increase the certainty of the target, and lead to a reduction of unnecessary PTV margins. To specifically reduce the respiratory movement, the use of a body compressor or shallow breathing technique with Abches is also applicable. In this study, such devices were used with the vertical movement of the respiratory diaphragm over $1 \mathrm{~cm}$, for selected patients who could tolerate them. Furthermore, 4D cone beam CT was available before delivery and this makes it possible for us to reduce the PTV margins $[24,25]$. Finally, the technique of real-time tumor tracking was applied to the trackable tumor using CyberKnife, which can reduce the safety margins [26]. Despite the small number of cases in our study, we found that patients who are treated with CyberKnife did not suffer from symptomatic RP, even though the PTVs, $V_{5}-V_{40}$ values, and small fraction numbers were comparable with those of the LINAC multiport group.

Additionally, we found that the rate of incidence of RP above grade 3 was very low (3\%) within 6 months after treatment with $S A B R$, and this rate is comparable to that observed in other studies $[27,28]$. When the rates of toxicities of over grade 3 are compared to other modalities of treatment such as surgery, SABR emerges as a potentially safer and more tolerable choice for patients. Chang et al. [1] reported the occurrence of grade 3-5 toxicities corresponding to treatment methods in stage I NSCLCs. In the group of patients treated with SABR, three patients (10\%) had grade 3 toxicities, two had dyspnea or cough, three had chest wall pain, and one had a rib fracture. In the group of patients that underwent 


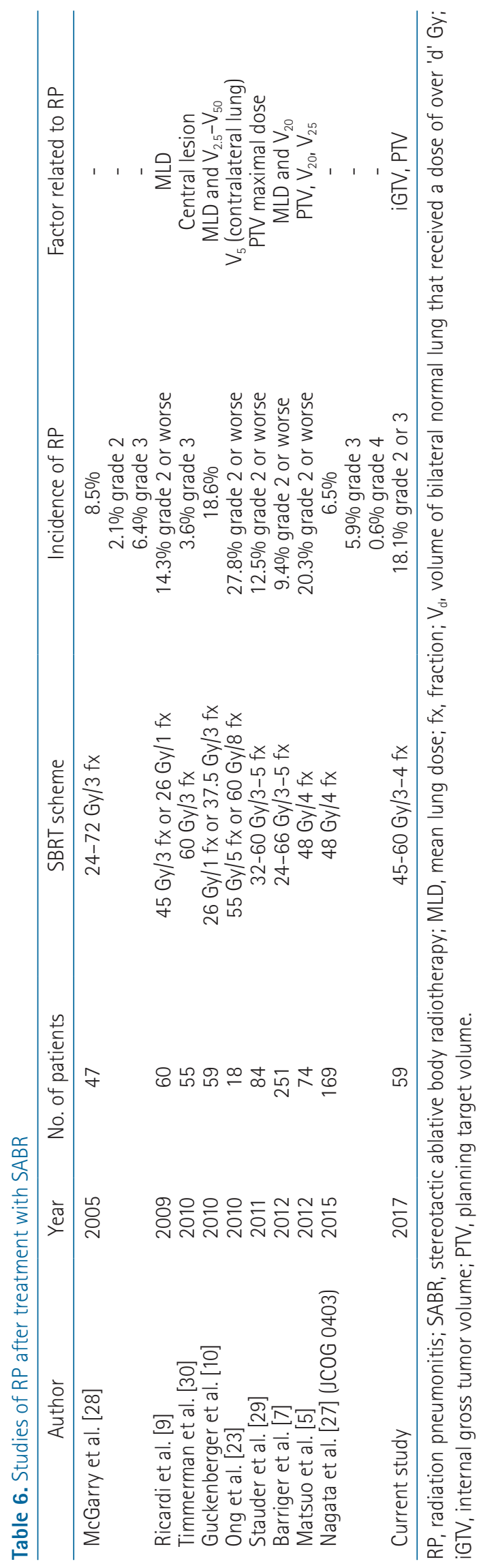

lobectomies, one patient died of surgical complications, and 12 (44\%) developed grade 3-4 toxicities related to treatment.

This study has some limitations. Firstly, the follow-up period in this study is short. However, it appears that a median follow-up period of 11 months is enough, upon the consideration that symptoms of RP generally appear at a median time of 5 months after treatment with SABR $[8,10]$ Secondly, deficient data on pulmonary function tests and preradiotherapy interventions may have compromised the quality of our data set. We could not suggest the performance of quantitative pulmonary function tests on patients before and after undergoing SABR because the pulmonary function test is not a mandatory examination for patients with metastatic tumors and for those who do not express discomfort or other symptoms. Thirdly, we lack data on patient previous thorax area surgery history which can damage lung parenchyme and induce fibrotic changes of lung. Thus our data cannot verify whether interventions or surgeries themselves influenced the development of RP.

In conclusion, the incidence of symptomatic RP following treatment with SABR was acceptable, with most patients showing RP of grade 1 or 2 ; there were no patients with grade 4 RP. Additionally, we found that both the large PTV and iGTV were predictive factors related to the development of symptomatic RP after treatment with SABR. Therefore, selected patients with small volumes of lung tumors, advanced $\mathrm{RT}$ techniques, such as respiratory gating and tracking systems, and appropriate dose prescriptions corresponding to tumor sizes are recommended for safer treatments with SABR. In the future, clinical trials with larger number of patients or metaanalyses of previous studies would be helpful for establishing the indications of the predictive factors that affect RP in lung tumors treated with SABR.

\section{Conflict of Interest}

No potential conflict of interest relevant to this article was reported.

\section{References}

1. Chang JY, Senan S, Paul MA, et al. Stereotactic ablative radiotherapy versus lobectomy for operable stage I non-smallcell lung cancer: a pooled analysis of two randomised trials. Lancet Oncol 2015;16:630-7.

2. Bertke MH, Bhatt NH, Hatch ME, Bousamra M, Van Berkel V, Dunlap NE. Comparative outcomes of stereotactic ablative 
radiation therapy (SABR) as salvage for parenchymal lung recurrences following initial curative surgery versus de-novo SABR. Int J Radiat Oncol Biol Phys 2015;93:E419-E420.

3. Timmerman R, McGarry R, Yiannoutsos $C$, et al. Excessive toxicity when treating central tumors in a phase II study of stereotactic body radiation therapy for medically inoperable early-stage lung cancer. J Clin Oncol 2006;24:4833-9.

4. Siva S, MacManus M, Ball D. Stereotactic radiotherapy for pulmonary oligometastases: a systematic review. J Thorac Oncol 2010;5:1091-9.

5. Matsuo Y, Shibuya K, Nakamura M, et al. Dose-volume metrics associated with radiation pneumonitis after stereotactic body radiation therapy for lung cancer. Int J Radiat Oncol Biol Phys 2012;83:e545-9.

6. Marks LB, Bentzen SM, Deasy J0, et al. Radiation dose-volume effects in the lung. Int J Radiat Oncol Biol Phys 2010;76(3 Suppl):S70-6.

7. Barriger RB, Forquer JA, Brabham JG, et al. A dose-volume analysis of radiation pneumonitis in non-small cell lung cancer patients treated with stereotactic body radiation therapy. Int J Radiat Oncol Biol Phys 2012;82:457-62.

8. Yamashita H, Nakagawa K, Nakamura $N$, et al. Exceptionally high incidence of symptomatic grade 2-5 radiation pneumonitis after stereotactic radiation therapy for lung tumors. Radiat Oncol 2007;2:21.

9. Ricardi U, Filippi AR, Guarneri A, et al. Dosimetric predictors of radiation-induced lung injury in stereotactic body radiation therapy. Acta Oncol 2009;48:571-7.

10. Guckenberger M, Baier K, Polat B, et al. Dose-response relationship for radiation-induced pneumonitis after pulmonary stereotactic body radiotherapy. Radiother Oncol 2010;97:65-70.

11. Borst GR, Ishikawa M, Nijkamp J, et al. Radiation pneumonitis in patients treated for malignant pulmonary lesions with hypofractionated radiation therapy. Radiother Oncol 2009;91:307-13.

12. Brown JM, Carlson DJ, Brenner DJ. The tumor radiobiology of SRS and SBRT: are more than the 5 Rs involved? Int J Radiat Oncol Biol Phys 2014;88:254-62.

13. Radiation Therapy Oncology Group. RTOG 0813: Seamless phase I/II study of stereotactic lung radiotherapy (SBRT) for early stage, centrally located, non-small cell lung cancer (NSCLC) in medically inoperable patients. Philadelphia, PA: Radiation Therapy Oncology Group; 2010.

14. Benedict SH, Yenice KM, Followill D, et al. Stereotactic body radiation therapy: the report of AAPM Task Group 101. Med Phys 2010;37:4078-101.
15. US Department of Health and Human Services. Common terminology criteria for adverse events (CTCAE) version 4.0. Bethesda, MD: National Cancer Institute; 2009.

16. Huang K, Dahele M, Senan $S$, et al. Radiographic changes after lung stereotactic ablative radiotherapy (SABR): can we distinguish recurrence from fibrosis? A systematic review of the literature. Radiother Oncol 2012;102:335-42.

17. Huang K, Palma DA; IASLC Advanced Radiation Technology Committee. Follow-up of patients after stereotactic radiation for lung cancer: a primer for the nonradiation oncologist. J Thorac Oncol 2015;10:412-9.

18. Hernando ML, Marks LB, Bentel GC, et al. Radiation-induced pulmonary toxicity: a dose-volume histogram analysis in 201 patients with lung cancer. Int J Radiat Oncol Biol Phys 2001;51:650-9.

19. Claude $L$, Perol D, Ginestet $C$, et al. A prospective study on radiation pneumonitis following conformal radiation therapy in non-small-cell lung cancer: clinical and dosimetric factors analysis. Radiother Oncol 2004;71:175-81.

20. Rancati T, Ceresoli GL, Gagliardi G, Schipani S, Cattaneo GM. Factors predicting radiation pneumonitis in lung cancer patients: a retrospective study. Radiother Oncol 2003;67:27583

21. Shi A, Zhu G, Wu H, Yu R, Li F, Xu B. Analysis of clinical and dosimetric factors associated with severe acute radiation pneumonitis in patients with locally advanced non-small cell lung cancer treated with concurrent chemotherapy and intensity-modulated radiotherapy. Radiat Oncol 2010;5:35

22. Ong CL. Volumetric modulated arc therapy for stereotactic body radiotherapy: planning considerations, delivery accuracy and efficiency [master's thesis]. Amsterdam: Vrije Universiteit; 2012.

23. Ong CL, Palma D, Verbakel WF, Slotman BJ, Senan S. Treatment of large stage I-II lung tumors using stereotactic body radiotherapy (SBRT): planning considerations and early toxicity. Radiother Oncol 2010;97:431-6.

24. Hof $H$, Rhein B, Haering $P$, Kopp-Schneider A, Debus J, Herfarth K. 4D-CT-based target volume definition in stereotactic radiotherapy of lung tumours: comparison with a conventional technique using individual margins. Radiother Oncol 2009;93:419-23.

25. Takahashi W, Yamashita $H$, Kida $S$, et al. Verification of planning target volume settings in volumetric modulated arc therapy for stereotactic body radiation therapy by using intreatment 4-dimensional cone beam computed tomography Int J Radiat Oncol Biol Phys 2013;86:426-31.

26. van der Voort van Zyp NC, Prevost JB, Hoogeman MS, et al. 
Stereotactic radiotherapy with real-time tumor tracking for non-small cell lung cancer: clinical outcome. Radiother Oncol 2009;91:296-300.

27. Nagata $Y$, Hiraoka $M$, Shibata $T$, et al. Prospective trial of stereotactic body radiation therapy for both operable and inoperable T1N0M0 non-small cell lung cancer: Japan Clinical Oncology Group Study JCOG0403. Int J Radiat Oncol Biol Phys 2015;93:989-96.

28. McGarry RC, Papiez L, Williams M, Whitford T, Timmerman RD. Stereotactic body radiation therapy of early-stage non-small- cell lung carcinoma: phase I study. Int J Radiat Oncol Biol Phys 2005;63:1010-5.

29. Stauder MC, Macdonald OK, Olivier KR, et al. Early pulmonary toxicity following lung stereotactic body radiation therapy delivered in consecutive daily fractions. Radiother Oncol 2011;99:166-71.

30. Timmerman R, Paulus R, Galvin J, et al. Stereotactic body radiation therapy for inoperable early stage lung cancer. JAMA 2010;303:1070-6. 